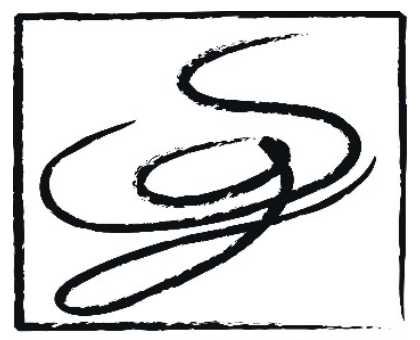

SEMINARIO DE INUESTIGACIÓN DE GÉNERO Y ESTUDIOS GULTURALES

\section{Fronteras cotidianas: intersecciones de espacios de exclusión y resistencia en una experiencia de migración desde América del Sur}

\author{
Everyday borders: intersections of \\ exclusion and resistance settings in a migration \\ experience from South America
}

\author{
Marina Calderón García \\ Universidad de Huelva \\ marinacaldegar@gmail.com
}

Fecha de recepción: 30/06//2021 Fecha de evaluación: 04/08/2021

Fecha de aceptación: 09/08/2021

\title{
Resumen
}

El presente trabajo tiene como objetivo reflexionar sobre los espacios de actividad cotidiana como generadores de exclusión y a la vez como disparadores para el desarrollo de la agencia y la transformación social y colectiva en contextos de migración. Las categorías de legitimidad creadas por las fronteras internacionales generan una brecha en el acceso a derechos de ciudadanía. Sin embargo, es en la interacción cotidiana donde con mayor amplitud se ponen de manifiesto una serie de fronteras simbólicas que dificultan la integración de la población migrante, más acentuado aun en el caso de las mujeres. A través del caso de Gladys, una mujer cuya experiencia migratoria involucra un viaje desde Perú hasta España, trataremos de ilustrar nuestro argumento y profundizaremos sobre los espacios de interacción a los que tiene acceso y cómo se generan una serie de fronteras simbólicas que modifican las relaciones entre las personas que habitan un territorio. Estas fronteras se superponen, creando un tejido de intersecciones que, si bien empujan hacia la exclusión social y el aislamiento, también suponen un espacio de desarrollo de la agencia. Concretamente, en el caso que presentamos en este trabajo, la experiencia fluctúa entre el ser considerada prescindible y a la vez ser una pieza fundamental para el sistema económico y social a través de los cuidados. Esta tensión se resuelve mediante el activismo y la reconfiguración de roles y prácticas. De esta forma nos invita a repensar el privilegio y el poder como 
procesos dinámicos y la capacidad de agencia individual, pero también comunitaria, como motor de la transformación política y social.

Palabras clave: migración; género; frontera simbólica; exclusión; agencia; feminismo interseccional; América del Sur

\begin{abstract}
The purpose of this paper is to reflect on everyday settings as drivers of both social exclusion in migrant contexts and resistance strategies. In such settings, migrants develop their agency and contribute to social transformations through individual and collective actions. International borders define the legitimacy of individuals and create a gap in terms of access to citizenship rights. However, it is in everyday interactions that symbolic borders pose the greatest hindrance to migrant integration. Such obstacles become even more evident for migrant women. This paper follows the journey of a migrant woman named Gladys from Peru to Spain, in an attempt to illustrate and discuss the social settings she is granted access to, and how symbolic borders arise that transform the relationships between people in those spaces. From an intersectional perspective, these boundaries collude to push migrants, particularly women, towards social exclusion and isolation. At the same time, these are also spaces where agency flourishes. Specifically, in Gladys' case as a caregiver, her experience fluctuates between being considered expendable labour and, at the same time, being a fundamental part of the economic and social fabric. This tension is eased through political activism and the reconfiguration of roles and practices. In this way, the case calls for a reflection on privilege and power as dynamic processes, on individual and communal agency as a main driver for political and social transformation.
\end{abstract}

Key words: migration; gender, symbolic border, exclusion, agency, intersectional feminism, South America

\title{
1. Introducción
}

El presente trabajo parte del contenido de la investigación desarrollada en el marco de una tesis doctoral en que abordaba la construcción de identidades en mujeres migrantes provenientes de América del Sur. En esta ocasión, con ayuda de uno de los casos, las reflexiones se dirigen hacia aquellos espacios generadores de exclusión en la experiencia migratoria y cómo en estas fronteras simbólicas se generan a su vez oportunidades para el cambio y la transformación individual y colectiva. Las migraciones, o movilidades, han sido y son un proceso de una gran complejidad. Son múltiples los factores que influyen en que una persona o colectivo acabe dejando su lugar de residencia habitual para continuar su vida en otro lugar. A su vez, son varias dimensiones las que se ponen en juego en este proceso. $Y$ por supuesto este proceso puede 
vivirse de muchas formas. No es la misma experiencia para un hombre que se desplaza desde Alemania para vivir en la Costa del Sol tras su jubilación que una mujer proveniente de Marruecos que trabaja como temporera en la recogida de fresa de Huelva. El nivel de implicación permitido en la comunidad o el reconocimiento de derechos no son tampoco similares en uno y otro caso. Por este motivo, cuando estudiamos sobre migraciones se hace necesario particularizar la o las experiencias sobre las que se está trabajando con el fin de no caer en generalizaciones que nos nublen la vista sobre las condiciones reales en la que se desenvuelven dichas vivencias individuales y colectivas.

Simplificando mucho el concepto de migración (United Nations 1998), podemos hablar de un criterio temporal sobre el que se establece el mínimo de un año para considerar que el cambio de residencia habitual se ha producido. A lo que hay que añadir que las migraciones pueden ser temporales, permanentes o incluso cíclicas si el desplazamiento entre los dos (o más) puntos geográficos se realiza de forma más o menos constante en el tiempo. Por otro lado, podemos hablar de movilidades individuales y de movilidades grupales o colectivas en función del número de personas que se embarcan en el mismo proyecto migratorio. También podemos hablar de migración al uso y migración de retorno en función de la relación previa que se mantuviera con el lugar de migración. Según un criterio espacial podemos encontrar migraciones internas si el desplazamiento se realiza dentro de las fronteras estatales, migraciones internacionales en el caso de que la migración traspase el Estado y migraciones intercontinentales en los casos en los que la población se mueve en un marco supranacional. Siendo esta una aproximación muy simple de las dimensiones sobre las que se puede abordar el proceso migratorio ya nos devuelve una imagen de fenómeno complejo y difícilmente homogeneizable.

A ello debemos sumar también la necesidad de una mirada de género y feminista sobre las migraciones, en las que se debe poner sobre la mesa las dimensiones políticas y de poder. De esta forma, entendemos el género como construcción social en la que los cuerpos leídos como mujeres son relegados a la periferia (Haraway 1995; Contreras Hernández y Trujillo Cristoffanini 2017).

Además de ser procesos con una gran diversidad intragrupo, los factores desencadenantes de las migraciones no son fruto de la casualidad ni de factores aleatorios. Con cierta frecuencia se considera que las migraciones dependen en exclusiva de una serie de decisiones de la o las personas que migran, perdiéndose de vista que forman parte de un proceso dentro de sistema global en el que políticas, economías y sociedades se encuentran interconectadas (Sassen 2013; De Genova 2016).

\section{Migraciones a Europa y España como país receptor}

La población total de personas migrantes provenientes de países no miembros de la Unión Europea o extracomunitarios supuso en 2019 casi el $5 \%(4,9 \%)$, es decir, en números absolutos se trata de 21,8 millones de personas. De esta cifra, el $71,3 \%$ se acumula entre Alemania, Italia, 
Francia y España. A estas cifras hay que sumar además, otros 13,3 millones de personas (un $3 \%$ de la población) que se desplazan dentro de Europa pero que cuentan con nacionalidad de alguno de los países de la Unión Europea (Eurostat 2021).

Europa lleva construyéndose como espacio sin fronteras internas de forma gradual desde 1985. Esto supone que las personas que ostentan la ciudadanía de cualquiera de los países pertenecientes a la Unión Europea tienen permitida la circulación de forma libre, así como un reconocimiento de derechos sociales como miembros de un mercado único. La construcción de una Europa sin fronteras internas tiene otras consecuencias en cuanto a las migraciones internacionales, supone por un lado el endurecimiento de las fronteras exteriores en lo que se ha denominado la Fortaleza Europa y por otro, la convergencia progresiva entre las políticas migratorias nacionales hacia un marco legal común (Sassen 2013; De Genova 2016; Burman 2005).

España forma parte del espacio Schengen de libre circulación de forma efectiva desde el año 1995 (desde 1986 ha sido miembro de la Unión Europea y desde 1999 miembro de la zona euro), pocos años antes de que comenzara a intensificarse la entrada de personas migrantes. Su localización geográfica la sitúa como frontera sur de Europa, lo que la convierte además en una de las puertas de acceso hacia la ya nombrada Fortaleza. Quizás también debido a su localización, a su experiencia con las migraciones circulares de trabajadores y trabajadoras temporales provenientes del Magreb, al paso relativamente rápido de país de emigración a país de inmigración o a sus condiciones estructurales, se ha ido desembocando en una visión utilitarista de la migración. (Sassen 2013; Izquierdo Escribano 2009). A pesar de que el título de la ley (Ley 4/2000) que regula las migraciones en España incluye la integración social, está claramente orientada a la migración laboral al vincular el permiso de residencia con la necesidad de contar con un contrato de trabajo de al menos un año para iniciar el trámite. Además, tras la crisis económica sujeta a una lenta recuperación, se suspendió durante varios años el presupuesto destinado a la integración de la población migrante. De esta forma, el esfuerzo principal se concentra en el control de acceso con la intención de no desestabilizar un mercado laboral nacional ya de por sí endeble. Esta situación contribuye a generar y mantener distintas condiciones laborales para personas comunitarias y extracomunitarias, es decir, un mercado de trabajo dual (Agrela 2002; Izquierdo Escribano 2009).

Como decíamos antes, las migraciones, sobre todo las laborales, son procesos pautados por un sistema económico progresivamente más global. Por este motivo, no es de extrañar que la pertenencia a un mercado común como el europeo pueda ser uno de los motivos principales para migrar a España. Además, la etapa de bonanza económica previa a la crisis supuso la necesidad de contar con mano de obra extranjera en sectores poco atractivos para la población autóctona, contribuyendo así a la creación de la imagen de España como país de inmigración. Se combina de esta forma un espacio de alta recepción de población migrante con una estructura débil en materia de extranjería y a la vez una estructura institucional debilitada 
que debe hacer frente al aumento progresivo del paro (Arroyo Pérez et al. 2014; Sassen 2013).

Con relación a los patrones geopolíticos que se establecen en los procesos de migración observamos que, restando a las personas provenientes de la eurozona, la afluencia principal para España de personas migrantes se vincula a zonas con las que tuvo un pasado colonial. América Latina supone entre un $25 \%$ y un $37 \%$ del total de flujo migratorio, con pesos diferenciados según país y momento histórico determinado. Concretamente, Ecuador, Colombia y Bolivia dominaron el volumen de migraciones de entrada durante los primeros años del siglo XXI, siendo las mujeres las protagonistas de estas movilidades (Arroyo Pérez et al. 2014; Izquierdo Escribano 2009).

De este pasado común de lazos coloniales con América Latina se derivan, además de una relación económica transnacional que se remonta siglos atrás, una mayor afinidad cultural producto de la propia colonización y una cierta flexibilidad en los requisitos para ingresar al territorio español, como la disminución del número de años de residencia necesarios para la solicitud de la nacionalidad, el reconocimiento de descendientes de personas exiliadas mediante la Ley de Memoria Histórica (Ley 52/2007) o la posibilidad de mantener la nacionalidad de origen tras la naturalización (Hierro 2016; OPAM 2016).

\section{Cultura, naciones $y$ fronteras}

Partimos de un contexto en el que el manejo de la diversidad previa a las migraciones es y ha sido complejo, ya sea en cuanto a la etnicidad, a la multinacionalidad y el plurilingüismo o en cuanto a la (a)confesionalidad del Estado. Sin embargo la negación de diversidad de la población española sin contar con el aporte de las migraciones contribuye a construir la idea de que la diferencia (la "otredad") viene de fuera de las fronteras nacionales, poniendo en una supuesta "distancia cultural" el peso para la dificultad para la integración (Zapata-Barrero 2009; Agrela 2002; Gregorio Gil 2009b).

Una vez que el flujo de migraciones se invierte y España pasa a ser un país de inmigración, se hace necesario que la gestión de la diversidad se extienda y atienda también con una lógica integrativa a aquellas personas que provienen de otros puntos geográficos. Cruzar la frontera dentro de los parámetros legales puede considerarse el primer paso para el reconocimiento progresivo de derechos, sin embargo, este dispositivo no garantiza la integración a niveles de acción cotidiana. Tal como indican los datos del MIPEX (Migrant Integration Policy Index) a pesar de contar con una buena puntuación general en cuanto a política migratoria en comparación con otros países del mundo, aún quedan algunas áreas con bastante margen de mejora como la educación, la participación política o las políticas antidiscriminatorias (Solano and Huddleston 2020). Por otro lado, existen diferencias regionales en cuanto al nivel de integración. La región sur, compuesta por Andalucía, Murcia, Ceuta y Melilla, presenta una alta densidad migratoria a la vez que unos indicadores socioeconómicos más débiles en comparación con regiones del norte, pero similares a grandes rasgos a nivel material entre la población autóctona y la población 
migrante. Sin embargo, debido al índice de precariedad y de informalidad laboral, las personas migrantes, y sobre todo las mujeres, se encuentran en una situación de mayor vulnerabilidad frente a las eventualidades como una crisis económica (Godenau et al. 2015; Agudelo-Suárez et al. 2013).

Resulta interesante pararse a observar cómo las fronteras parecen funcionar a modo de límites simbólicos donde se generan categorías culturales asociadas a la nacionalidad con alta consistencia interna y fácilmente distinguibles entre sí. Por ejemplo, las categorías "extranjero", "marroquí" o "boliviana". De acuerdo con Alonso (2008) y con Burman (2005) la cultura se convierte en esencia cuando se confunde con la soberanía sobre un territorio, convirtiendo de esta forma a los grupos nacionales en homogéneos. En este sentido la distancia cultural a la que apelábamos al inicio de este apartado recae sobre comunidades imaginadas entendidas de manera ahistórica, en lugar de entenderse como construcciones recientes propias del paradigma de modernidad (Mignolo 2007).

En un sentido más profundo, Kearney (2008) otorga a las fronteras una doble función. Por un lado, la función clasificatoria que facilita la inclusión o exclusión de ciertas categorías como el género, la etnia, o la clase social. Por otro lado, cumple una función de transformación del valor clase a través de la cual se redefinen a nivel estructural las interacciones posibles. De esta forma, la categoría "extranjero" expondría a posiciones estructurales de mayor vulnerabilidad y explotación dentro del mercado laboral. Así, y de acuerdo con Beck (2006), el foco de atención debería moverse de estas concepciones homogeneizadoras a puntos de tensión y transformación en la experiencia. Es decir, a la práctica de la acción cotidiana, a los escenarios y los discursos en los que se tejen y sin perder de vista el sistema global capitalista en el que esas prácticas se han ido conformando históricamente (Glick Schiller, Basch y Blanc-Szanton 1992; Bauman 2002).

En la misma línea, la opinión pública sobre la inmigración (Centro de Investigaciones Sociólogicas 2017) pone de manifiesto que es en los aspectos que tocan la interacción cotidiana como la sanidad, educación, trabajo o libertad religiosa donde mayor displicencia muestra la sociedad receptora. Sin embargo, aparentemente, no parece existir un rechazo explícito hacia la entrada y la permanencia de personas migrantes. Aunque conocidas son las condenables demostraciones de xenofobia relacionadas con los hechos de muestras de El Ejido en el año 2000 (Constenla and Torregrosa 2000), Elche en 2004 (Cachón Rodríguez 2012) o El Tarajal en 2014 (Sánchez 2014). Por otro lado, incluso son percibidos como un factor de enriquecimiento cultural en un sentido amplio, apuntando de esta manera a una concepción más heterogénea y en constante transformación de los grupos humanos.

\section{Fronteras simbólicas, interseccionalidad y mujeres migrantes}

Una vez que hemos puesto en cuestión la idea de cultura como sinónimo de nación, nos falta ampliar el concepto teniendo en cuenta que las dimensiones de género, sexualidad o clase, al igual que cualquier tipo de mediación con el entorno natural o social también forman parte del 
mismo. De esta forma, la cultura se compone de infinidad de escenarios y prácticas en las que cada persona puede estar inmersa y que devienen en experiencias y formas de estar en el mundo únicas.

Volviendo al tema de las migraciones, la consideración unidimensional de la experiencia a través de la cuestión racial o colonial no contribuye a la comprensión de la complejidad que implican. De este modo, se hace necesario tomar prestada la noción de interseccionalidad, que nos permite ampliar a la par que particularizar las condiciones en las que se desarrolla el proceso migratorio a través de la articulación de escenarios, prácticas, significados y discursos, así como las relaciones de poder que se establecen en las interacciones a distintos niveles. La interseccionalidad es una herramienta que nos permite atravesar nociones universalizadoras, sea la adscripción a una nacionalidad, por ejemplo "peruana", o categorías como "migrante" y "mujer", de forma que en vez de comprenderse al margen del contexto de producción, se integren en una relación múltiple, simultánea y mutuamente constructiva a través de la propia experiencia situada históricamente (Gregorio Gil 2009c; Viveros Vigoya 2016; Van Meijl 2012; Burman 2005).

Con esta visión interseccional podemos entender, por ejemplo, que la separación entre los espacios públicos y privados propia de nuestra experiencia occidental responde a una estructura cultural que separa los espacios propios correspondientes a hombres y mujeres. Así, el espacio que define a las mujeres es el privado. Este espacio privado de cuidados se aleja de las percepciones laborales de la sociedad y por tanto no se considera remunerable. Sin embargo, sobre ellas recae la obligación de la reproducción biológica y social y por tanto se erigen como guardianas de los valores morales y nacionales. Se entiende, de esta forma, por qué el control sobre las mujeres de su sexualidad y su reproducción tenga tanto peso (Burman 2005).

De acuerdo con Gregorio Gil (2009a, 2017) las mujeres migrantes, precisamente por el hecho de tener esta doble condición de ser mujeres y ser migrantes, se enfrentan a una serie de fronteras simbólicas que las constituye como un grupo homogéneo. Por ejemplo, se espera de ellas que traerán al mundo una amplia descendencia, lo cual desde posiciones conservadoras supone poner en peligro la identidad europea de una ciudadanía legítima. Esta ciudadanía legítima se entiende desde dichas posiciones conservadoras en términos de blanquitud en relación con una supuesta raza caucásica, valores cristianos y elementos simbólicos compartidos a través de una identidad nacional colectiva. Frente a una representación de las mujeres migrantes desde la otredad casi barbárica. Igualmente, a las mujeres migrantes se las asocia a dos escenarios laborales relacionados con la dicotomía de la sexualidad femenina desde un discurso heteropatriarcal, es decir, se les asocia a la prostitución o a la reproducción social mediante los cuidados y el servicio doméstico en beneficio de las mujeres occidentales.

Las mujeres migrantes se enfrentan además a un discurso que trata de despojarlas de agencia y las encasilla en los términos de pobreza, analfabetismo, irregularidad y victimismo, además de considerar que los 
espacios de desarrollo de actividad laboral que les son propios son la prostitución o el servicio doméstico (Gregorio Gil 2009c). Como veremos en un momento posterior, podemos detectar un vínculo entre las medidas políticas tomadas desde el gobierno, la construcción del discurso de migración a través de los medios, la opinión social y la situación de exclusión a la que se enfrentan las mujeres migrantes, que, sin embargo, no queda exenta de respuesta por su parte, ya que estas mujeres ponen en marcha mecanismos de acción y resistencia frente a la lógica de la expulsión social.

\section{Un caso de experiencia de migración desde América del Sur}

Para llevarlo al terreno de lo concreto, traemos aquí el caso de Gladys que nos permitirá poder en relación los elementos que hemos ido desarrollando hasta ahora y cómo se configuran dentro de una experiencia particular de migración. Como ya señalábamos al inicio del presente trabajo, este caso pertenece a la investigación desarrollada con motivo de una tesis doctoral en la que se establecieron los cimientos para estudiar la construcción de la identidad dialógica en contextos migratorios a través del análisis de narraciones de mujeres migrantes provenientes de América del Sur. Para el desarrollo de la investigación doctoral nos basamos principalmente en una perspectiva dialógica de la identidad (Hermans 2001b; Aveling, Gillespie y Cornish 2015) y en las propuestas metodológicas del Análisis Crítico del Discurso (Wodak et al. 2003; Lazar 2007) y del Análisis de la Enunciación (Larraín y Medina 2007).

\subsection{Descripción del caso}

Gladys llegó a Sevilla (España) en el año 2007 con 32 años desde su tierra natal en Chosica (Perú). Su entrada fue de forma regular, por lo que contaba con contrato de trabajo en el momento de cruzar la frontera. Su motivación para migrar sin embargo no fue laboral, sino que pretendía reunirse con su pareja que había tomado la iniciativa unos cuantos años antes, contando de esta forma con una pequeña red de acogida desde sus inicios.

Gladys cuenta con otra experiencia de migración internacional previa, durante la cual residió en Argentina, donde sí ocupó un lugar central la motivación económica a la hora de emigrar, para ayudar a su familia de origen en su situación financiera. En los años anteriores a la migración Gladys finalizó en Perú estudios superiores relacionados con la mercadotecnia dedicando posteriormente su actividad laboral a la administración pública local. En cambio, tras venir a España el nicho laboral que ha ocupado desde su llegada ha sido el de servicio doméstico. Su situación económica personal se ha visto resentida debido a las condiciones laborales y empeorada sensiblemente tras la crisis económica que sufrió el país desde 2008, viéndose abocada a recurrir a servicios ofrecidos por instituciones que asisten a personas en riesgo de exclusión social. 


\subsection{Escenarios relacionados con su experiencia migratoria}

A través de su entrevista, en la que hicimos un recorrido por los eventos más importantes de su vida y revisamos su experiencia migratoria, podemos destacar una serie de escenarios en los que tiene sus principales interacciones como mujer migrante, sean éstas de forma directa o indirecta. Entre ellos además veremos que se producen una serie de intersecciones en las que se producen negociaciones de significados a través de los discursos presentes en las propias interacciones (Hermans 2001b; Aveling, Gillespie y Cornish 2015; Bhatia 2002).

En primer lugar, el escenario familiar es uno de los escenarios relevantes en lo que se refiere a la experiencia migratoria de Gladys por ser donde se encuentra su principal red de apoyo. Esta red está compuesta por, además de ella misma, su padre, su madre, su abuela, sus hermanos y hermanas, sus cuñados y cuñadas, un hermano menor por parte de padre a nivel biológico (aunque criado también por la madre de Gladys), su marido, el hijo biológico de su marido (y adoptado como hijo propio por ella) y su suegra. Se trata de un escenario transnacional, pues se compone de personas que residen tanto en España como en Perú y aun así debe considerarse de interacción directa y cercana. En este escenario se incluye también a la Iglesia Católica por su influencia en la articulación del discurso sobre la familia que presenta el caso de Gladys.

A continuación, el escenario educativo toma un papel preponderante en la toma de decisiones que acompañaron el proceso migratorio de Gladys. Esto se debe por un lado a la importancia que ella misma le confiere a la educación y por otro, a la dificultad para el reconocimiento de su titulación académica tras la migración, aun así, ella decidió continuar con el proceso con la idea de acompañar a su pareja. De nuevo encontramos un escenario transnacional en el que aparecen como participantes antes de la migración además de la propia Gladys, sus padres, el personal de administración de la Universidad en la que realizó sus estudios en Perú y que le asesoraron en el proceso de reconocimiento, al igual que uno de sus profesores, y posteriormente una vez establecida en España, su hijo Luis y su marido.

El escenario laboral surge seguidamente a lo largo de su narración. Como ya dijimos en su presentación, a pesar de contar con una titulación universitaria y experiencia en la administración pública en Perú, tras realizar la migración su actividad se focaliza en el servicio doméstico. En España, la regulación de la permanencia está vinculada al contrato de trabajo (Ley $4 / 2000$, Real Decreto 557/2011), por lo que la negociación de este requisito con el empleador o empleadora se hace indispensable para mantener la condición de regularidad. Recordamos que Gladys vino a España de forma regular y por tanto con un contrato de trabajo en origen, también en el sector de cuidados como empleada de hogar. Es uno de los escenarios que más interacciones sociales presenta puesto que representa un eje central en la articulación de su experiencia. Así, cuenta con la presencia de su marido y su cuñada, varios compañeros y compañeras de profesión, diversas 
personas que cumplen el papel de empleador/a, una amiga y otras personas migrantes.

Por otro lado, el escenario sanitario surge de forma específica en la narración de Gladys para tratar el tema del trato desigual con el que se atiende a las personas migrantes. En esta ocasión, el escenario se enmarca en la sociedad de acogida. Sobre todo, aparecen referencias al personal administrativo del centro de salud al que Gladys está adscrita. Además de la relación con las instituciones médicas, también se recogen particularidades de su estado de salud y el de su familia.

Por el contrario, el escenario de apoyo institucional emerge como espacio donde encontrar recursos para paliar su difícil situación económica familiar. Al igual que el escenario anterior, también se encuentra relacionado con los momentos posteriores a la migración. En este escenario aparecen instituciones de la administración pública como el área social del Ayuntamiento, instituciones pertenecientes a la Iglesia Católica como Cáritas y otras personas migrantes que pueden ejercer como beneficiarias de ciertas ayudas y también proporcionar información sobre las mismas. Cada organización genera su propio escenario concreto con normativa reguladora específica donde se sientan las bases que generan distintos tipos de acción e interacción.

Cercano al escenario laboral, aunque, como veremos en el siguiente apartado, en respuesta al mismo se encuentra el escenario político. La participación en este escenario está mediada por la Oficina de Derechos Sociales de Sevilla ${ }^{1}$. A partir de los primeros contactos con dicha entidad y poniendo en relación su experiencia con la de otras personas en situación similar, Gladys comienza a tomar conciencia de la precariedad estructural concomitante al sector del servicio doméstico. El centro del escenario lo ocupa la acción de lucha por los derechos laborales de las personas que trabajan como empleadas de hogar. También se trata de un escenario posterior a la migración conformado por la Oficina de Derechos Sociales de Sevilla, una abogada colaboradora y compañeras y compañeros de la organización.

A otro nivel de participación no tan directa y no de forma excesivamente explícita, aparece en la narración de Gladys el escenario legislativo. Encontramos por un lado referencias a la regulación de las condiciones laborales y su vinculación con los requisitos para la permanencia legal en España, y por otro lado encontramos referencias en relación al acceso para personas migrantes a servicios como el de salud, así como la homologación de las titulaciones universitarias. Aunque no aparecen actores específicos en este escenario, se puede entender como principal interlocutor al Estado.

Por último, consideramos un escenario global, que hemos denominado escenario migratorio, en el que convergen y se articulan los escenarios anteriores, que han ido surgiendo a través del relato de Gladys, en conjunto con la coyuntura económica, social e institucional del momento. Este escenario será el objeto de análisis del siguiente apartado.

\footnotetext{
1 Colectivo conformado por una gran variedad de agentes provenientes de distintos ámbitos que trabajan contra la precariedad y en favor de derechos sociales
} 
5.3. Intersecciones de espacios de exclusión y resistencia en una experiencia de migración

A continuación, se presentan algunos fragmentos de la entrevista2 en la que Gladys detalló su experiencia migratoria y que permitirán ilustrar nuestro argumento. La narración de Gladys se articula en torno a dos posiciones principales en continua tensión que se han construido a través de las intersecciones entre distintos escenarios . De esta forma, encontramos un deslizamiento entre el ser prescindible y el ser sostén para la vida.

En cuanto a la primera de ellas, la noción de ser prescindible se sustenta en el discurso de xenofobia construido a través de las intersecciones entre el escenario legislativo y los escenarios laboral, educativo y sanitario, generándose una idea migración como mano de obra y una lógica de expulsión de los escenarios cuando se considera que la mano de obra ya no es necesaria. Así, por ejemplo, la aprobación por parte del Estado del Real Decreto-Ley 16/20123 que limitaba el sistema universal de salud y por tanto restringía el acceso a los servicios sanitarios para las personas migrantes en situación irregular se sustentaba en la insostenibilidad de las arcas públicas tras la crisis económica de 2008. De una forma bastante contundente la intersección entre el escenario legislativo y el sanitario establece una categorización y jerarquización de personas, las que pueden y las que no pueden ser atendidas en la sanidad pública. Esta circunstancia empeoró la situación de muchas personas que se vieron privadas de este servicio encontrándose de partida en una situación de desventaja.

G134: "La crisis ha sido fuerte ¿no? O sea, m: los primeros en cierta manera, pasarlo mal hemos sido los inmigrantes ¿no?"

Pero la cosa no quedó ahí, sino que se extendió en algunos casos como el de Gladys a personas que se encontraban en situación de regularidad y que veían dificultado el acceso a los servicios por parte del personal de administración de los centros, de los que dependían para ello. Ante esta situación Gladys se ve en la situación de tener que defender la legitimidad de su petición a través de los requisitos legales de permanencia.

G12: "Pedí consulta y me negaron la atención de la consulta porque dice que yo: mi tarjeta estaba caducada y digo "no: está por caducar" y luego yo le: yo le digo "pero vamos," "no: es que ahora la política, no se puede atender" y le digo "pero vamos

\footnotetext{
${ }^{2}$ Material disponible para su consulta

${ }^{3}$ Actualmente se encuentran derogados varios de sus efectos a través del Real DecretoLey $7 / 2018$, sin embargo, aún se encontraba plenamente vigente en el momento de realizar la entrevista

${ }^{4}$ La entrevista de Gladys (G) se dividió en secciones temáticas, el número indica la seriación correlativa de dichas secciones
} 
espérate, yo soy no soy inmigrante ilegal sin papeles, o sea, vamos que: soy inmigrante sí". En 2012, en 2012 estaba con la cabeza fuera de aquí. Yo me acuerdo que tenía la tarjeta, yo era legal, con tarjeta de residencia y mi tarjeta de residencia estaba por vencerse ¿vale? Pero estaba dentro de plazo, y ya había presentado el expediente en Plaza en España como se debe hacer. Tres meses antes"

Otra de las intersecciones que sustenta la posición de ser prescindible es la que se da entre los escenarios legislativo, educativo y laboral. Por un lado, debido a cuestiones administrativas, el no poder homologar su titulación universitaria, Gladys se ve en la situación de aceptar trabajos en peores condiciones, en cuanto a precariedad e informalidad, para poder optar a tener y mantener el permiso de residencia. Dichas condiciones a su vez funcionan como barrera para la promoción laboral hacia otros nichos mejor valorados. Tal como indica la Ley Orgánica 4/2000, de 11 de enero, sobre Derechos y Libertades de los Extranjeros en España y su Integración Social, es requisito contar con un contrato de trabajo de al menos un año de duración para poder solicitar el permiso de residencia. En este sentido, la legitimidad o no de la permanencia parece depender de las personas que actúan como empleadoras, que pueden llegar a imponer condiciones difíciles para llegar firmar el contrato laboral o suponer un obstáculo a modo de frontera simbólica a la hora de que personas migrantes puedan ocupar puestos de mayor reconocimiento social:

G6: "Ahora en cuanto a la parte laboral (.) e:h (.) yo desde que he llegado: he limpiado casa ¿sabes? [...] En el trabajo en el que yo estoy hay mucha: ¿qué te digo? mucho abuso en cuanto: la gente hace lo que quiere. Yo trabajo: ¿en cuántas casas? Martes y viernes voy a una casa, (.) el jueves voy a dos casas, el miércoles voy a una casa. No sé cuántas son, 4 . Vale. Estoy dada de alta en una sola. [El empleador] es pepero y trabaja en: un cargo alto. $Y$ por eso ha sido que me ha dado de alta. Los demás no. Ni se lo piensan, vamos. Y una de las excusas que me ponen es que no es que no: que los gastos del gestor y todo lo demás [...] Pero también e:h un poco por la situación de la crisis. Mi esposo no está trabajando, este año está con la prestación. E:h pues yo decía bueno qué hago, le digo sus cuatro cosas y me quedo sin trabajo ¿sabes? Entonces es eso. No es tanto el miedo. Miedo es de no: de repente: no encontrar otro trabajo. Porque en realidad digo si es que: el mercado laboral e:h de España o de Sevilla estaba cuando yo llegué, cuando yo llegué, yo le daba el lujo de decir no o de decir (.) te dejo ¿no? Y. y buscar otra porque había oferta ¿no? O sea, m: el trabajo que tú tienes es una bendición y tienes que cuidarlo porque no hay"

G7: "Y aquí hay que demostrar la valía mucho más. Si eres extranjero ¿no? Para: poder tener un trabajo ¿no?", "Y yo digo, bueno, yo lo veo un poco difícil el buscar, pero bueno lo voy a seguir intentando." 
En cuanto a los prejuicios, que también funcionan como barreras, a los que Gladys se enfrenta en el escenario laboral, son de muy diversos y se muestran de forma explícita en relación con la representación social del nivel de estudios de las personas migrantes y la homogeneización de rasgos físicos, cuestión que no queda sin respuesta por parte de Gladys:

G6: "Y: y tiene mucho prejuicio. Dice la gente de aquí. Que piensan que la gente que viene de América Latina, (.) m: no sé yo lo digo para viajar de Perú a España hay un filtro. O sea: la gente no viene ilegal de Perú a España ¿qué tiene que hacer? Debe tener un contrato de trabajo o tiene que ser por lo menos, conocer ciertos aspectos, que no cualquiera lo hace ¿no? Entonces la señora con la que yo trabajaba me preguntaba si yo sabía leer. Entonces, bueno tengo un título universitario y no sé leer. $Y$ después me dijo que todos tenemos los mismos rasgos. O sea, "todos ustedes, las bolivianas, ecuatorianas, tienen los mismos rasgos" digo claro descendemos todos de ustedes [risa] o sea: que son cosas como te digo (.) tontas ¿no?"

Se producen también prejuicios en cuanto a la preferencia hacia personas de origen nacional frente a las migrantes para ocupar puesto de trabajo en un contexto de crisis económica, de forma que se refuerza la idea de que existen personas legítimas y no legítimas o aceptadas y rechazadas:

G7: "Yo el trabajo de administrativa que tuve, mi: mi: amiga, pues tuvo un problema ahí porque sus compañeros le decían "oye que tienes gente aquí: que: ¿por qué metes a tus amigas?" o sea, vamos, se jugó mucho. Yo también sé que se jugó mucho por nosotras. Por apostar por nosotras. Para que trabajemos ahí"

A la par y en contraposición a la posición de prescindible surge la posición de ser sostén de la vida dentro de un sistema de cuidados familista. Esto significa que su papel dentro de la estructura es fundamental para que el sistema económico y social siga funcionando. Esta posición se sustenta en dos niveles a partir de su empleo dentro del sector de servicio doméstico. Por un lado, a nivel microscópico por ser la entrada económica principal en su núcleo familiar en un mercado de trabajo que, como se ha podido entrever en algunos fragmentos anteriores, se entiende dividido en ocupaciones para hombres y mujeres:

G6: "Ahora en cuanto a la parte laboral (.) e:h (.) yo desde que he llegado: he limpiado casa ¿sabes?"

G14: "Porque yo creo que la: el mercado laboral de varones en España todavía está un poco: difícil. Más si eres inmigrante, 
porque él [el marido] sí tenía problemas. Trabajaba de guarda: en una comunidad"

Y por otro lado a nivel macroscópico puesto que su función como cuidadora y limpiadora en las familias que la emplean como asalariada supone la liberación de carga de trabajo a sus miembros (principalmente a las mujeres) y por tanto permite que sus miembros dispongan de tiempo para la formación o para otras cuestiones que pueden mejorar su calidad de vida. Se aumenta de esta forma la situación de privilegio de partida para las familias locales y se genera una brecha en la carga de cuidados aún mayor:

G11: "[...] A decir "mira, que nosotras llevamos un trabajo indispensable para la vida, o sea, muy muy importante." $\mathrm{Y}: \mathrm{y}$ eso hay que que yo eso se lo digo a mis compañeras "tú créetelo, o sea, realmente créetelo," no es porque tú digas que tu trabajo es lo: lo: es que realmente es importante, es que: $\mathrm{m}$ : hay personas que: yo cuido niños, por ejemplo, y yo sé que la señora que la señora si: yo no voy a cuidarle los niños ahora por la tarde, por ejemplo, ella no podría ir al médico con el otro niño, o no podría hacer muchas cosas. Entonces e:h como que: frenaría muchas cosas en la sociedad. Hay que: yo digo hay que empoderarse ¿no? Y eso, o sea, vamos, que tengo pa' más [risa]"

En respuesta a esta tensión entre ser considerada a la vez como mano de obra prescindible y como parte fundamental de un sistema de cuidados que se basa en la familia, Gladys encuentra una salida en la acción colectiva a través de su participación en la Oficina de Derechos Sociales de Sevilla (ODS):

G7: "A raíz de la situación (.) que sería lo que es el empleo de hogar, pues me: (2) desde ya más de 3, 4 años, que estoy participando en un grupo, de la ODS. Y que en cierta medida cambió mi manera de pensar ¿vale?"

G9: "Eso es un poquito que me hizo que: que mi actitud cambiara ¿no? A través de la ODS yo empecé en estas reuniones"

G15: "Entonces yo siento que ese cambio, me ha llevado a mí a abrir mi mente ¿no' llegar aquí y dejar de ser la niña consentida, asumir una familia, ver otra realidad, trabajar de: de oficina a trabajar de chacha (.) es duro ¿no?"

Sin embargo, como veremos a continuación, la participación en el escenario político no solo le ha permitido erigirse como agente de transformación en el escenario laboral, sino también en cuanto a las relaciones de género en el escenario familiar. Así, en relación al escenario laboral Gladys toma conciencia de lo improcedente de su situación laboral al contrastarla con una abogada de la propia organización después de que 
su marido hiciera referencia a las condiciones laborales de servicio doméstico, relacionadas con el sueldo, previas a la crisis:

G8: "O sea, solo nos decían nuestros derechos y ahí quedaban ¿no? Y no sé cómo un día voy a una de estas charlas y veo a las chicas de la ODS ¿no? Y: una de estas abogadas de este: yo decido ir con ella para que me explique un poco más sobre mi situación. Entonces me dice "esto es así, tu contrato debe de ser así, tu esto y tu nómina también" "iah! ¡Qué interesante!" entonces dije estos hijos de puta me están [risa] a parte que yo lo sabía, porque mi esposo me decía que, en el tiempo de él, cuando él llegó, la hora no: la hora se pagaba mínimo 7 euros. Y ahora me querían pagar hasta 5. [...]"

Gladys toma conciencia de que es tratada como simple mano de obra explotable en un nicho laboral muy poco valorado económica y socialmente y en el que se le solicita que cumpla funciones, más allá de la limpieza, relacionadas con el bienestar que no son reconocidas ni por los empleadores ni por el Estado:

G8: "[...] Encima te piden: que hagas de todo, porque aquí parece que de empleada de hogar tuvieras una profesión. No: no: la sirvienta, sino una profesión. Cuidar niños, enfermería, de jardinería, que seas manitas ¿qué más? No sé, hasta me han hecho limpiar paredes."

G9: "Yo decía yo llevo bienestar, porque yo de alguna manera me tengo que desconectar de los problemas que tengo en mi casa, para escuchar el rollo de la señora que me está hablando y todo y claro yo tengo que empatizar con ella y la señora se siente liberada, se siente feliz conmigo. Claro yo estoy trabajando y hablando y escuchando la historia y "qué bien me escuchas y que bien me entiendes" ¿no? Y yo me enrollo con ella y claro y luego llego a mi casa y digo claro, yo tengo un poco de terapia porque tengo que escuchar a todas ellas. $Y$ eso. [...]"

Por otro lado, en cuanto al escenario familiar y las relaciones de género que allí se construyen, Gladys parte de un discurso de género segregado en cuanto a tareas y obligaciones fundamentado sobre todo en las enseñanzas de su abuela rodeadas de un entorno más bien cerrado y ratificadas posteriormente por su marido:

G9: “[...] nos enseñaron en Perú, mi abuela ¿no? Tenía esa idea muy fuerte. La mujer es para la casa. Y a mujer que está en la calle, esa es una mujer vaga porque primero tiene que ver que su casa esté limpia, que su cocina esté limpia y si no hay nada que hacer en casa, siempre hay algo que hacer, que hay que coser, que hay que planchar, que hay y que: toda mujer tiene 
que prepararse para eso, o sea, que tiene que saber planchar, que tiene que saber cocinar, que tiene que saber coser [...]"

G10: "Yo cuando me casé no sabía ni planchar, tanto es así que mi esposo para que yo planche bien me compró una vaporella "para que no sufras planchando" [risa]"

G15: "De repente por la situación en la que yo vivía, por cómo me habían educado ¿no? Por donde me desarrollaba y todo lo demás. M: la Iglesia era parte de nuestra vida y todo lo demás, pero: no veía más allá ¿no?”

En contraposición, tras su experiencia personal, laboral y política comienza a fraguar una serie de cambios hacia un discurso más igualitario, aunque sin salir del discurso binarista y segregado de hombre-mujer. Se trata de una toma de conciencia que apela no solo a su capacidad de agencia individual sino también a su capacidad de transformación como parte de un colectivo:

G9: "[...] como dice mi esposo "te has vuelto feminista" ¿no?"

G10: "luego aprendí y luego le dije "no, que la casa la hacemos los dos" y la casa la llevamos los dos. Y que la casa no es mía y que la responsabilidad de la casa no es mía, que él actualmente limpia la casa. Cuando puede ¿no? Él limpia la casa, él cocina y: él hace la compra, porque yo detesto hacer la compra.", "no está de más que haya igualdad entre mujeres y hombres. Y que el trato sea igual. Igualdad de oportunidades en todo ¿no? Que si yo quiero un trabajo que es netamente hombre, que lo puedo conseguir. Que nada me limita a eso ¿no? Entonces e:h actualmente esa transformación, yo digo que si me vieran en Perú: como soy ahora se diría "¡madre! Que esta es tu: Porque de repente en Perú tenía una cultura muy: sumisa, muy: como decía, muy de derechas, ahora soy muy de izquierdas, muy de pensar en empoderar a la mujer, muy de pensar en reivindicar mis derechos, o sea, estoy participando es más liderando el grupo de empleadas de hogar, o sea, prácticamente me he autoproclamado la portavoz del grupo. Porque antes entre las mismas chicas no: entonces bueno dije yo tomo la iniciativa, porque nadie: nadie en sí, entonces yo decía no, yo tomo la iniciativa y el grupo es asociación actualmente ¿no?"

G15: "Mira yo en Perú era: una chica de clase media, católica, (.) como decir tú eres pepera [risa]. Yo digo, bueno, lo voy a reconocer, yo: tenía un pensamiento de derechas, de repente no era tanto de izquierdas como lo soy ahora ¿no?".

Como vemos en el caso de Gladys, su experiencia como mujer migrante supone una configuración compleja de situaciones que la llevan en un sentido a situarse en el límite de lo aceptado y en otro sentido como parte sustancial del sistema social y económico. Gladys realiza un recorrido que parte de posiciones de aceptación de la situación y sumisión a la norma 
cultural para acabar reivindicándose como agente en un proceso de transformación individual y colectiva.

\section{Conclusiones}

El objetivo del presente trabajo se ha centrado en reconocer que en las migraciones internacionales no solo es necesario cruzar una frontera física que delimita un territorio nacional. Más allá de esta cuestión administrativa, la vida de las personas se desarrolla en torno a una serie de prácticas culturales situadas en escenarios que pueden delimitarse de forma más o menos explícita pero que tienen sus normas de interacción. Para las personas migrantes el acceso a la participación en estos escenarios puede suponer la superación de barreras cotidianas o fronteras simbólicas que dificultan la integración o un trato igualitario respecto a la población autóctona.

Como hemos visto en el caso de Gladys, además, los escenarios pueden interseccionar entre sí haciendo más compleja la experiencia migratoria. De esta forma, y de acuerdo con Kearney (2008) en estos cruces de fronteras sucede una transformación del valor estructural, reduciendo en este caso la consideración de ser humano a mano de obra útil. Es decir, al cruzar fronteras se genera un espacio negado de pertenencia donde se ubica la otredad y donde se restringen derechos de ciudadanía (Mignolo, 2007), a la par que se homogeneiza a la población migrante apelando a cuestiones principalmente nacionales (Martínez, 1988).

Así, podemos encontrar una frontera clara en la legitimidad de acceso a servicios básicos como el de sanidad o educación, bien sea a través de dispositivos como normativas o de interacciones con desigualdad de poder. Igualmente, el acceso a puestos de trabajo con buenas condiciones queda limitado por la necesidad de obtener un contrato de trabajo que dé acceso a un permiso de residencia. Además, la distancia de separación que actúa como frontera se ve reforzada por ejemplo a través de la apelación a los rasgos físicos o a una supuesta diferencia en el nivel educativo asociadas a la nacionalidad en un discurso de tintes xenófobos (Stolcke 1995).

Si a esto añadimos la cuestión de género, veremos cómo el campo de acción se cierra sobre actividades relacionadas con el cuidado y el servicio doméstico. De esta forma se impone otra frontera más a superar derivada de la alta demanda de mujeres inmigrantes, sobre todo de origen latinoamericano, para este tipo de puestos. Además, tiene como efecto generar cadenas globales de cuidado que traspasan fronteras transnacionales y se basan en una desigualdad estructural entre las propias mujeres, encontrándose la mujer que cuida en una posición de mayor vulnerabilidad que la que recibe el servicio de cuidado (Oso y Parella 2012; Gregorio Gil 2010).

Sin embargo como hemos visto en el caso de Gladys, lejos de proponer una perspectiva victimizadora, se hace necesario hacer hincapié en la capacidad de agencia y por tanto en la acción para sobreponerse a estos límites y ejercer como agentes frente al resto de la ciudadanía a 
través de la reivindicación e incluso transformación de derechos individuales y colectivos (Sassen 2013; Castles y Davidson 2000). Como por ejemplo, en la reclamación del reconocimiento para el empleo más allá del sector de cuidados o la negociación de los roles y tareas dentro del hogar (Amorós Domínguez y Contreras Hernández 2017; Oso y Parella 2012).

Por tanto, se hace necesario particularizar y situar contextualmente las experiencias de migración desde una perspectiva interseccional, sin perder de vista los marcos de referencia global. Del mismo modo se deben tener en cuenta los procesos dinámicos de poder a los que dichas experiencias están sujetas, de forma que pueda apreciarse en qué espacios se producen las barreras y sus consecuentes transformaciones de significado y la reorganización de la práctica cotidiana (Viveros Vigoya 2016; Gergen y Gergen 2006).

En cuanto a cuestiones pendientes que se plantean en este trabajo se propone lo siguiente. Puesto en ningún caso se puede extrapolar ni generalizar las conclusiones sobre la experiencia migratoria de mujeres a partir de un solo caso, se podría ampliar el número y la diversidad de los mismos de forma que nos permitieran contrastar las experiencias y ampliar y profundizar sobre cómo se enfrentan las mujeres migrantes a las fronteras cotidianas. Además, sería interesante tratar el tema de la construcción de género dentro de la migración como contenido específico de las entrevistas más que como fondo, de forma que se suscitara la reflexión explícita. Por último, también sería de interés contribuir a la deconstrucción del concepto de "mujer migrante", muy sesgado desde la óptica heteropatriarcal. Este reto podría conseguirse a través de la inclusión en el estudio de una mayor diversidad de experiencias subjetivas en torno a las relaciones afectivas 0 identidades sexuales.

\section{Referencias bibliográficas}

AGRELA, Belén. "La Política de Inmigración En España: reflexiones sobre la emergencia del discurso de la diferencia cultural." Migraciones Internacionales 1 (2): 93-121, 2002.

AGUDELO-SUÁREZ, Andrés A., et al. "Impact of Economic Crisis on Mental Health of Migrant Workers: What Happened with Migrants Who Came to Spain to Work?" International Journal of Public Health 58 (4): 627-31, 2013.

ALONSO, Ana María. "Borders, Sovereignty, and Racialization." In A Companion to Latin American Anthropology, edited by Deborah Poole, 230-52. New Jersey: Blackwell Publishing, 2008.

AMORÓS DOMÍNGUEZ, Màrius y CONTRERAS HERNÁNDEZ, Paola Andres. "Agencia Femenina En Los Procesos Migratorios Internacionales: Una Aproximación Epistemológica." Empiria, no. 37: 75-99, 2017.

ARROYO PÉREZ, Andrés, BERMÚDEZ PARRADO, Silvia, ROMERO VALIENTE Juan Manuel, HERNÁNDEZ RODRÍGUEZ, Juan Antonio y PLANELLES ROMERO, Joaquín. Una Aproximación Demográfica a La Población Extranjera En España. Madrid: Ministerio de Empleo y Seguridad Social, 2014.

AVELING, Emma-Louise, GILLESPIE Alex y CORNISH, Flora. "A Qualitative 
Method for Analysing Multivoicedness." Qualitative Research 15 (6): 670-87. https://doi.org/10.1177/1468794114557991, 2015.

BAUMAN, Zygmunt. La Cultura Como Praxis. Barcelona: Editorial Paidós. 2002. BECK, Ulrich. The Cosmopolitan Vision. Cambridge: Polity Press, 2006.

BHATIA, Sunil. "Acculturation, Dialogical Voices and the Construction of the Diasporic Self." Theory \& Psychology 12 (1): 55-77. https://doi.org/0803973233, 2002.

BURMAN, Erica. "Engendering Culture in Psychology." Theory \& Psychology 15 (4): 527-48. https://doi.org/10.1177/0959354305054750, 2005.

CACHÓN RODRÍGUEZ, Lorenzo. "Bases Sociales de Los Sucesos de Elche de Septiembre de 2004: Crisis Industrial, Inmigración y Xenofobia." Edited by Subdirección General de Información Administrativa y Publicaciones (Institución). Madrid, 2012.

CASTLES, Stephen y DAVIDSON, Alastair. "The Crisis of Citizenship." In Citizenship and Migration. Globalization and the Politics of Belonging, 1-25. Houndmills, Basingstoke, Hampshire and London: Macmillan Press, 2000.

CENTRO DE INVESTIGACIONES SOCIÓLOGICAS. "Actitudes Hacia La Inmigración(X)." Madrid. http://www.cis.es/cis/export/sites/default/Archivos/Marginales/3180_3199/3190/es3190mar.pdf., 2017.

CONSTENLA, Tereixa y TORREGROSA, Ana. "Vecinos de El Ejido Armados Con Barras de Hierro Atacan a Los Inmigrantes y Destrozan Sus Locales." El País, $1-8$.

https://elpais.com/diario/2000/02/07/espana/949878022_850215.html. 2000.

CONTRERAS HERNÁNDEZ, Paola y TRUJILLO CRISTOFFFANINI, Macarena. "Desde Las Epistemologías Feministas a Los Feminismus Decoloniales: Aportes a Los Estudios Sobre Migraciones." Athenea Digital 17 (1): 145-62. https://doi.org/10.5565/rev/athenea.1765, 2017.

EUROSTAT. Migrant Integration Statistics - 2020 Edition. Edited by Katarzyna Kraszewska, Piotr Juchno, and Ani Todorova. Luxembourg: Publications Office of the European Union, 2021.

GENOVA, Nicholas P. De. 'The 'Crisis' of the European Border Regime: Towards a Marxist Theory of Borders." International Socialism, n. 150: 33-56. http://isj.org.uk/the-crisis-of-the-european-border-regime-towards-a-marxisttheory-of-borders/, 2016.

GERGEN, Mary M. y GERGEN, Kenneth J. "Narratives in Action." Narrative Inquiry 16 (1): 112-21. https://doi.org/10.1075/ni.16.1.15ger, 2006.

GLICK SCHILLER, Nina, BASCH, Linda y BLANC-SZANTON, Cristina. "Transnacionalism: A New Analytic Framework for Understanding Migration." Annals of the New York Academy of Sciences 645: ix-xi: 1-24. https://doi.org/10.1111/j.1749-6632.1992.tb33482.x. 1992.

GODENAU, Dirk, RINKEN, Sebastián, MARTÍNEZ DE LIZARRONDO ARTOLA, Antidio y MORENO MÁRQUEZ, Gorka. "La Integración de Los Inmigrantes En España: Una Propuesta de Medición a Escala Regional." Documentos Del Obeservatorio Permanente de La Inmigracion, Vol. 30. Madrid, 2015.

GREGORIO GIL, Carmen. 2009a. "Fronteras de Género y Cultura En El Contexto de La Fortaleza Europa." In I Congreso Internacional Género y Frontera, 104:42-54. San Cristóbal de La Laguna.

GREGORIO GIL. 2009b. "Mujeres Inmigrantes: Colonizando Sus Cuerpos Mediante Fronteras Procreativas, Étnico-Culturales, Sexuales y Reproductivas." Viento Sur, no. 104: 42-54.

GREGORIO GIL. 2009c. "Silvia, ¿quizás Tenemos Que Dejar de Hablar de Género y Migraciones? Transitando Por El Campo de Los Estudios Migratorios." Gazeta de Antropología 25 (1): 1-17. 
GREGORIO GIL. 2010. “Debates Feministas En El Análisis de La Inmigración No Comunitaria En El Estado Español. Reflexiones Desde La Etnografía y La Antropología Social." Relaciones Internacionales 14: 93-116.

GREGORIO GIL. 2017. "Etnografiar Las Migraciones 'Sur'-'Norte': La Inscripción En Nuestros Cuerpos de Representaciones de Género, Raza y Nación." Empiria, no. 37: 19-39.

HARAWAY, Donna J. "Conocimientos Situados: La Cuestión Científica En El Feminismo y El Privilegio de La Perspectiva Parcial." In Ciencia, Cyborgs y Mujeres. La Reinvención de La Naturaleza, edited by Donna J. Haraway, 313-46. Madrid: Cátedra, 1995.

HERMANS, Hubert J.M. 2001a. "The Construction of a Personal Position Repertoire: Method and Practice." Culture \& Psychology 7 (3): 323-66. https://doi.org/10.1177/1354067X0173005.

HERMANS, Hubert. 2001b. "The Dialogical Self: Toward a Theory of Personal and Cultural Positioning." Culture \& Psychology 7 (3): 243-81. https://doi.org/10.1177/1354067X0173001.

HIERRO, María. "Latin American Migration to Spain: Main Reasons and Future Perspectives." International Migration 54 (1): 64-83. https://doi.org/10.1111/imig.12056, 2016.

IZQUIERDO ESCRIBANO, Antonio. "En La Antesala de La Recesión: Inmigración y Modelo Inmigratorio." El Modelo de Inmigración y Los Riesgos de Exclusión. Madrid, 2009.

JEFATURA DEL ESTADO. 2000. Ley Orgánica 4/2000, de 11 de Enero, Sobre Derechos y Libertades de Los Extranjeros En España y Su Integración Social. España.

JEFATURA DEL ESTADO. 2011. Real Decreto 557/2011, de 20 de Abril, Por El Que Se Aprueba El Reglamento de La Ley Orgánica 4/2000, Sobre Derechos y Libertades de Los Extranjeros En España y Su Integración Social, Tras Su Reforma Por Ley Orgánica 2/2009.

KEARNEY, Michael. "La Doble Misión de Las Fronteras Como Clasificadoras y Como Filtros de Valor." In Migraciones, Fronteras e Identidades Étnicas Transnacionales, edited by Laura Velasco Ortiz, 79-116. Tijuana: El Colegio de la Frontera Norte and Miguel Ángel Porrúa, 2008.

LARRAÍN, Antonia y MEDINA, Lorena. "Análisis de La Enunciación: Distinciones Operativas Para Un Análisis Dialógico Del Discurso." Estudios de Psicología 28 (3): 283-301. https://doi.org/10.1174/021093907782506443, 2007.

LAZAR, Michelle M. "Feminist Critical Discourse Analysis: Articulating Feminist Discourse Praxis." Critical Discourse Studies 4 (2): 141-64, 2007.

MARTÍNEZ, Oscar J. "Mexican Americans, Ethnic Conflict, and Identity Issues." In Troublesome Border, 76-99. Tucson, Arizona: The University of Arizona Press, 1988.

MEIJL, Toon Van. "Multiculturalism, Multiple Identifications and the Dialogical Self: Shifting Paradigms of Personhood in Sociocultural Anthropology." In Handbook of Dialogical Self Theory, edited by Hubert J.M. Hermans and Thorsten Gieser, 98-114. New York: Cambridge University Press, 2012.

MIGNOLO, Walter D. La Idea de América Latina. La Herida Colonial y La Opción Decolonial. Barcelona: Editorial Gedisa, 2007.

OBSERVATORIO PERMANENTE ANDALUZ DE LAS MIGRACIONES (OPAM). "Informe Bienal OPAM 2014-15." Sevilla, 2016.

OSO, Laura y PARELLA, Sònia. "Inmigración, Género y Mercado de Trabajo : Una Panorámica de La Investigación Sobre La Inserción Laboral de Las Mujeres Inmigrantes En España." Cuaderno de Relaciones Laborales 30 (1): 11-44, 2012. 
SÁNCHEZ, Gabriela. "Las Muertes de Ceuta: ¿Quién Ha Mentido?" EIDiario.Es, 1-8. https://www.eldiario.es/desalambre/mentiras-giran-alrededor-muertesceuta_1_5024093.html. 2014.

SASSEN, Saskia. Inmigrantes y Ciudadanos. De Las Migraciones Masivas a La Europa de La Fortaleza. Madrid: Siglo XXI. 2013.

SOLANO, Giacomo y Thomas Huddleston. Migrant Integration Policy Index 2020. Barcelona-Brussels: CIDOB and MPG. 2020

STOLCKE, Verena. "Talking Culture: New Boundaries, New Rhetorics of Exclusion in Europe." Current Anthropology 36 (Special Issue: Ethnographic Authority and Cultural Explanation): 1-24. https://doi.org/10.1086/204339. 1995.

UNITED NATIONS. "Recommendations on Statistics of International Migration." New

York. https://unstats.un.org/unsd/publication/SeriesM/SeriesM_58rev1E.pdf\%0Aht tp://www.armstat.am/file/doc/99475948.pdf. 1998.

VIVEROS VIGOYA, Mara. "La Interseccionalidad: Una Aproximación Situada a La Dominación." Debate Feminista 1-17. https://doi.org/10.1016/j.df.2016.09.005. 2016.

WODAK, Ruth, MEYER, Michael, JÄGER, Siegfried, VAN DIJK, Teun A., FAIRCLOUGH, Norman y SCOLLON, Ron. Métodos de Análisis Crítico Del Discurso. Edited by Ruth Wodak and Michael Meyer. Barcelona: Editorial Gedisa, 2003.

ZAPATA-BARRERO, Ricard.. "Dynamics of Diversity in Spain: Old Questions, New Challenges." In The Multiculturalism Backlask. European Discourses, Policies and Practices, edited by Steven Vertovec and Susanne Wessendorf. London: Routledge, 2009. 